CBPF-NF-054/94

DFTT-045/94

\title{
The Quantum Algebraic Structure of the Twisted XXZ Chain
}

\author{
M.R-Monteiro ${ }^{1, a}$, I. Roditi ${ }^{1, b}$, L.M.C.S. Rodrigues ${ }^{1, c}$ and S. Sciuto ${ }^{2, d}$ \\ ${ }^{1}$ Centro Brasileiro de Pesquisas Físicas - CBPF \\ Rua Dr. Xavier Sigaud, 150 \\ 22290-180 Rio de Janeiro, RJ, Brasil \\ ${ }^{2}$ Dipartimento di Fisica Teorica dell' Universitá di Torino \\ and Sezione di Torino dell' INFN, Via P. Giuria 1, I-10125, Torino, Italy
}

\begin{abstract}
We consider the Quantum Inverse Scattering Method with a new R-matrix depending on two parameters $q$ and $t$. We find that the underlying algebraic structure is the twoparameter deformed algebra $S U_{q, t}(2)$ enlarged by introducing an element belonging to the centre. The corresponding Hamiltonian describes the spin-1/2 XXZ model with twisted periodic boundary conditions.
\end{abstract}

e-mail addresses:
(a)mmont@cbpfsu1.cat.cbpf.br
(b) roditi@cbpfsu1.cat.cbpf.br
(c) ligia@brlncc.bitnet
(d) sciuto@to.infn.it 
Many completely integrable one-dimensional quantum models have been treated [1] by the Quantum Inverse Scattering Method (QISM) 2, 3] which, among other achievements, led to the discovery of Quantum Groups [4] independently from Drinfeld and Jimbo [5]. In this letter we use this method to describe the relation between the spin- $1 / 2 \mathrm{XXZ}$ chain with twisted periodic boundary conditions and the two-parameter deformed algebra $S U_{q, t}(2)$ enlarged by introducing an element belonging to the centre, showing that the second parameter of the deformation, $t$, is linked to the twist.

The QISM introduces an auxiliary problem with the help of the so-called Lax operator. In our model this operator is

$$
L_{n}(\lambda, t)=\left(\begin{array}{cc}
t^{Z_{n}-S_{n}^{3}} \operatorname{sh}\left[\gamma\left(\lambda+i S_{n}^{3}\right)\right] & i S_{n}^{-} \sin \gamma \\
i S_{n}^{+} \sin \gamma & t^{-Z_{n}-S_{n}^{3}} \operatorname{sh}\left[\gamma\left(\lambda-i S_{n}^{3}\right)\right]
\end{array}\right),
$$

where $\vec{S}_{n}$ and $Z_{n}$ are operators defined on the $n-t h$ vectorial space of the periodic $\left(\vec{S}_{N+1} \equiv \vec{S}_{1}, Z_{N+1} \equiv Z_{1}\right)$ chain, which in the fundamental representation are given by

$$
Z_{n}=\frac{1}{2} 1_{n}, \vec{S}_{n}=\frac{1}{2} \vec{\sigma}_{n}
$$

where $\vec{\sigma}$ are the Pauli matrices and $\mathbb{l}$ is the identity operator.

The $R$-matrix associated to the Lax operator $(1)$ is

$$
R(\lambda, t)=\left(\begin{array}{cccc}
a(\lambda) & 0 & 0 & 0 \\
0 & c^{\prime}(\lambda) & b(\lambda) & 0 \\
0 & b(\lambda) & c^{\prime \prime}(\lambda) & 0 \\
0 & 0 & 0 & a(\lambda)
\end{array}\right),
$$

where

$$
\begin{aligned}
& a(\lambda)=\operatorname{sh}[\gamma(\lambda+i)] \\
& b(\lambda)=i \sin \gamma \\
& c^{\prime}(\lambda)=t c(\lambda) \\
& c^{\prime \prime}(\lambda)=t^{-1} c(\lambda)
\end{aligned}
$$


and

$$
c(\lambda)=\operatorname{sh} \gamma \lambda
$$

clearly, $R(\lambda)=R(\lambda, t=1)$ is the appropriate matrix for the XXZ model [6]. It is easy to check that the matrix $R(\lambda, t)$ (eqs. (3-5)) satisfies the Yang-Baxter equation [7, 8]

$$
R_{12}\left(\lambda_{12}, t\right) R_{13}\left(\lambda_{13}, t\right) R_{23}\left(\lambda_{23}, t\right)=R_{23}\left(\lambda_{23}, t\right) R_{13}\left(\lambda_{13}, t\right) R_{12}\left(\lambda_{12}, t\right)
$$

and that $L_{n}(\lambda, t)$ (eq. (1)) obeys the Fundamental Commutation Relations $(F C R)$

$$
R_{12}\left(\lambda_{12}, t\right) L_{n}^{1}\left(\lambda_{1}, t\right) L_{n}^{2}\left(\lambda_{2}, t\right)=L_{n}^{2}\left(\lambda_{2}, t\right) L_{n}^{1}\left(\lambda_{1}, t\right) R_{12}\left(\lambda_{12}, t\right)
$$

In (6) and (7), $\lambda_{i j}=\lambda_{i}-\lambda_{j}$ and

$$
\begin{aligned}
& R_{12}=\sum_{i} a_{i} \otimes b_{i} \otimes 1 \quad, \quad R_{13}=\sum_{i} a_{i} \otimes 1 \otimes b_{i}, \\
& R_{23}=\sum_{i} 11 \otimes a_{i} \otimes b_{i},
\end{aligned}
$$

with the $R(\lambda, t)$ matrix written as

$$
R(\lambda, t)=\sum_{i} a_{i} \otimes b_{i}
$$

and the upper indices in eq. (7) follow

$$
L^{1}=L \otimes 1 \quad, \quad L^{2}=11 \otimes L .
$$

We notice that the $R$-matrix is defined on the tensor product of two auxiliary spaces $\mathbb{C}^{2} \otimes \mathbb{C}^{2}$ and the $L$-matrix is defined on the tensor product of the auxiliary space $\mathbb{C}^{2}$ and the internal space $\mathbb{C}^{d}$, with $d$ the dimension of the representation of the associated algebra satisfied by the operators in the elements of the matrix $L$.

The reason for $R(\lambda, t)$ to satisfy eq. (6) is that it can be written in terms of $R(\lambda)$ as

$$
R_{12}(\lambda, t)=g^{1}\left(g^{2}\right)^{-1} R_{12}(\lambda) g^{1}\left(g^{2}\right)^{-1}
$$

\footnotetext{
${ }^{1}$ Equivalently one could write

$$
g^{1}\left(g^{2}\right)^{-1}=t^{S^{3} \otimes Z-Z \otimes S^{3}},
$$

with $S^{3}$ and $Z$ given by eq. (2). This form is more appropriate if one wishes to compare the algebraic structure here presented with ref. [9]. In a forthcoming paper we shall discuss this subject, as well as the relationship of our approach with the one in ref. [10].
} 
where

$$
g^{1}=g \otimes 1 \quad, \quad g^{2}=1 \otimes g \quad, \quad g=t^{\frac{1}{2} S^{3}}
$$

and

$$
\left[g^{1} g^{2}, R_{12}(\lambda)\right]=0
$$

Moreover, eq. (7) follows from eq. (6), because (for $\mathrm{d}=2$ )

$$
L_{n}(\lambda, t)=R_{o, n}\left(\lambda-\frac{i}{2}, t\right)
$$

where "o" labels the auxiliary space. We also observe that

$$
R(0, t)=P
$$

where $P$ is the permutation operator on the tensor product of the two spaces where the $R$-matrix is defined.

According to the standard procedure of the QISM, eq. (7) allows one to build an infinite set of commuting operators

$$
F(\lambda, t)=\operatorname{Tr}\left[L_{N}(\lambda, t) \cdots L_{2}(\lambda, t) L_{1}(\lambda, t)\right]
$$

where both the matrix product and the trace are performed in the auxiliary space.

In our case the Bethe Ansatz equations for the fundamental representation are given by

$$
\left(\frac{\alpha\left(\lambda_{\beta}\right)}{\delta\left(\lambda_{\beta}\right)}\right)^{N}=t^{-N} \prod_{\substack{\alpha=1 \\ \alpha \neq \beta}}^{M}\left\{\frac{a\left(\lambda_{\beta}-\lambda_{\alpha}\right)}{a\left(\lambda_{\alpha}-\lambda_{\beta}\right)} \frac{c\left(\lambda_{\alpha}-\lambda_{\beta}\right)}{c\left(\lambda_{\beta}-\lambda_{\alpha}\right)}\right\} ; \beta=1, \cdots, M \leq N
$$

with

$$
\begin{aligned}
& \alpha(\lambda)=\operatorname{sh}\left[\gamma\left(\lambda+\frac{i}{2}\right)\right] \\
& \delta(\lambda)=\operatorname{sh}\left[\gamma\left(\lambda-\frac{i}{2}\right)\right]
\end{aligned}
$$

explicitly showing the contribution due to the parameter $t$, as $\alpha, \delta, a$ and $c$ are the same functions appearing in the XXZ model. 
In order to show the algebraic structure underlying the $R$ and $L$ matrices defined in eqs. (1) and (3-5) we perform a suitable similarity transformation [3] on (6) and (7) which permits us to have the following decomposition:

$$
\begin{aligned}
& \tilde{L}_{n}(\lambda, t)=\frac{1}{2}\left(e^{\lambda \gamma} L_{+}-e^{-\lambda \gamma} L_{-}\right) \\
& \tilde{R}\left(\lambda_{i j}, t\right)=e^{\gamma \lambda_{i j}} R_{+}-e^{-\gamma \lambda_{i j}} R_{-} ;
\end{aligned}
$$

where

$$
\begin{aligned}
& L_{+}=\left(\begin{array}{cc}
q^{S^{3}} t^{Z-S^{3}} & \Omega S^{-} \\
0 & q^{-S^{3}} t^{-Z-S^{3}}
\end{array}\right) \\
& L_{-}=\left(\begin{array}{cc}
q^{-S^{3}} t^{Z-S^{3}} & 0 \\
-\Omega S^{+} & q^{S^{3}} t^{-Z-S^{3}}
\end{array}\right)
\end{aligned}
$$

and

$$
R_{+}=\left(\begin{array}{cccc}
q & 0 & 0 & 0 \\
0 & t & \Omega & 0 \\
0 & 0 & t^{-1} & 0 \\
0 & 0 & 0 & q
\end{array}\right),
$$

with $\Omega=q-q^{-1}$ and $R_{-}=P R_{+}^{-1} P$.

Substituting eq. (19) in the Y-B equation (6) and in the FCR, eq. (7), one gets the following independent equations:

$$
\begin{aligned}
& R_{+} L_{\varepsilon}^{1} L_{\varepsilon}^{2}=L_{\varepsilon}^{2} L_{\varepsilon}^{1} R_{+} \quad(\varepsilon= \pm 1) \\
& R_{+} L_{+}^{1} L_{-}^{2}=L_{-}^{2} L_{+}^{1} R_{+},
\end{aligned}
$$

which imply that the operators in the entries of $L$ must satisfy

$$
\begin{aligned}
& {\left[S^{3}, Z\right]=\left[S^{ \pm}, Z\right]=0} \\
& {\left[S^{3}, S^{ \pm}\right]= \pm S_{ \pm}} \\
& t^{-1} S^{+} S^{-}-t S^{-} S^{+}=t^{-2 S^{3}}\left[2 S^{3}\right]_{q}
\end{aligned}
$$


where $[x]_{q}=\left(q^{x}-q^{-x}\right) /\left(q-q^{-1}\right)$ with $q=\exp (i \gamma)$. Eqs. (23) are the commutation relations of the two-parametric deformed $\mathrm{SU}(2)$ [9, 11, 12] with $Z$, an element of the center of the resulting algebra. The coproduct is obtained by considering the product of two $L_{\varepsilon}$ acting on two internal spaces and we find:

$$
\begin{aligned}
& \Delta S^{3}=S^{3} \otimes 1+1 \otimes S^{3} \\
& \Delta Z=Z \otimes 1+1 \| Z \\
& \Delta S^{ \pm}=q^{S^{3}} t^{\mp Z-S^{3}} \otimes S^{ \pm}+S^{ \pm} \otimes q^{-S^{3}} t^{ \pm Z-S^{3}} .
\end{aligned}
$$

The coproduct $(24 \mathrm{c})$ is related to the one in ref. [12 by a similarity transformation generated by the operator $t^{S^{3} \otimes Z-Z \otimes S^{3}}$.

Following the QISM, a local Hamiltonian can be written as

$$
\left.H \propto \frac{\partial}{\partial \lambda} \ell g F(\lambda, t)\right|_{\lambda=\frac{i}{2}}
$$

and thanks to eqs. (14-16), it becomes for the fundamental representation of the algebra

$$
\begin{aligned}
& H=\sum_{i=1}^{N} H_{i, i+1} \quad(N+1 \equiv 1) \\
& H_{i, i+1}=\left.\frac{J \sin \gamma}{i \gamma} \frac{\partial}{\partial \lambda} \hat{R}_{i, i+1}(\lambda, t)\right|_{\lambda=0},
\end{aligned}
$$

where $\hat{R}=P R$ and $R(\lambda, t)$ is given by eqs. (3-5). In the above equation $R_{i, i+1}(\lambda, t)$ acts on the two internal spaces $(i, i+1)$ instead of acting on two auxiliary spaces.

Substituting $R(\lambda, t)$ given by eqs. (3-5) in eq. (26), apart from an additive constant, we get

$$
H=\frac{J}{2} \sum_{i=1}^{N}\left[2 t^{-1} \sigma_{i}^{+} \sigma_{i+1}^{-}+2 t \sigma_{i}^{-} \sigma_{i+1}^{+}+\frac{q+q^{-1}}{2} \sigma_{i}^{z} \sigma_{i+1}^{z}\right],
$$

where $\sigma^{ \pm}=\left(\sigma^{x} \pm i \sigma^{y}\right) / 2$.

Such a Hamiltonian is very similar to the XXZ model with periodic boundary conditions but for each pair of sites $(i, i+1)$, the site $(i+1)$ is rotated of an angle $\alpha\left(t=e^{i \alpha}\right)$ in the $x-y$ plane with respect to the site $i$. 
The similarity transformation generated by $\exp \left\{-i \frac{\alpha}{2} \sum_{\ell=1}^{N}(\ell-1) \sigma_{\ell}^{z}\right\}$ takes the Hamiltonian (eq. (27)) to

$$
\begin{aligned}
H= & \frac{J}{2}\left[\sum_{n=1}^{N-1}\left(\sigma_{n}^{x} \sigma_{n+1}^{x}+\sigma_{n}^{y} \sigma_{n+1}^{y}+\cos \gamma \sigma_{n}^{z} \sigma_{n+1}^{z}\right)+\right. \\
& \left.\cos \gamma \sigma_{N}^{z} \sigma_{1}^{z}+2 t^{-N} \sigma_{N}^{+} \sigma_{1}^{-}+2 t^{N} \sigma_{N}^{-} \sigma_{1}^{+}\right],
\end{aligned}
$$

which is the well-known [13] Hamiltonian for the XXZ chain with twisted periodic boundary conditions.

It is amusing to observe that, thanks to eq. (13) and following the procedure of ref. [10], the Hamiltonian (eq. (27)) could also be obtained from the $R$-matrix of the XXZ model $R(\lambda)=R(\lambda, t=1)$, using $L_{n}^{\prime}(\lambda, t)=t^{S^{3}} L_{n}(\lambda, t=1) \neq L_{n}(\lambda, t)$. Conversely, the

untwisted XXZ model can be built from $R(\lambda, t)$ (eqs. $(3-5))$ and $L_{n}^{\prime \prime}(\lambda, t)=t^{-S^{3}} L_{n}(\lambda, t)$. All these topics will be discussed in detail in a forthcoming paper.

Finally, we would like to point out that by introducing a central element $Z$ which enlarges the $S U_{q, t}(2)$ algebra, we make appear the underlying algebraic structure of the so-called twisted XXZ model.

\section{Acknowledgements}

S. Sciuto thanks CBPF for its kind hospitality and CNPq for financial support. I. Roditi thanks D. Altschuler for useful discussions. 


\section{References}

[1] P. Kulish and E. Sklyanin, Lect. Notes in Phys. 151 (1982) 61 (and ref. therein);

N. Andrei, "Integrable Models in Condensed Matter Physics", ICTP Summer Course, Trieste, 1992 (and ref. therein);

V. Korepin and F. E $\beta$ ler, "Exactly Solvabe Models of Strongly Correlated Electrons" (and ref. therein), cond-mat/9408092.

[2] E. Sklyanin, L. Takhtajan and L. Faddeev, Teor. Matem. Fiz. 40 (1979) 194;

L. Takhtajan and L. Faddeev, Russian Math. Surveys 34 (1979) 11.

[3] L. Faddeev, in "New Problems, Methods and Techniques in Quantum Field Theory and Statistical Mechanics", edited by M. Rasetti, World Scientific Pub. (1990); "Algebraic Aspects of Bethe-Ansatz", preprint ITP-SB-94-11.

[4] P. Kulish and N. Reshetikhin, Zap. Nauch. Seminarov LOMI 101 (1981) 101; J. Sov. Math. 23 (1983) 2435.

[5] V. Drinfeld, Sov. Math. Dokl. 32 (1985) 254;

M. Jimbo, Lett. Math. Phys. 10 (1985) 63; 11 (1986) 247.

[6] E. Lieb, Phys. Rev. Lett. 18 (1967) 692; Phys. Rev. 162 (1968) 162; Phys. Rev. Lett. 18 (1967) 1046; Phys. Rev. Lett. 19 (1967) 108.

[7] R. Baxter, Ann. of Phys. 70 (1972) 193; Ann. of Phys. 70 (1972) 323.

[8] C.N. Yang, Phys. Rev. Lett. 19 (1967) 1312.

[9] N. Reshetikhin, Lett. Math. Phys. 20 (1990) 331.

[10] H.J. de Vega, Nucl. Phys. B240 (1984) 495; Int. J. Mod. Phys. A4 (1989) 2371.

[11] A. Sudbery, J. Phys. A23 (1990) L697; M. Takeuchi, Proc. Jap. Acad. 66 (1990) 112. 
[12] $\breve{C}$. Burdik and L. Hlavatý, J. Phys. A24 (1991) L165.

[13] F. Alcaraz, U. Grimm and V. Rittenberg, Nucl. Phys. B316 (1989) 735;

V. Pasquier and H. Saleur, Nucl. Phys. B330 (1990) 523. 